\title{
Assessment of Transient Stability through Coherent Machine Identification by Using Least-Square Support Vector Machine
}

\author{
Bhanu Pratap Soni $\mathbb{D}^{1},{ }^{1}$ Akash Saxena $\mathbb{D D}^{2}$ Vikas Gupta $\mathbb{D D}^{1}$ and S. L. Surana $\mathbb{D}^{2}$ \\ ${ }^{1}$ Department of Electrical Engineering, Malaviya National Institute of Technology, Jaipur, Rajasthan 302017, India \\ ${ }^{2}$ Department of Electrical Engineering, Swami Keshvanand Institute of Technology, Jaipur, Rajasthan 302017, India \\ Correspondence should be addressed to Bhanu Pratap Soni; er.bpsoni2011@gmail.com
}

Received 23 December 2017; Revised 20 March 2018; Accepted 29 March 2018; Published 10 May 2018

Academic Editor: Elio Chiodo

Copyright (c) 2018 Bhanu Pratap Soni et al. This is an open access article distributed under the Creative Commons Attribution License, which permits unrestricted use, distribution, and reproduction in any medium, provided the original work is properly cited.

\begin{abstract}
Transient stability assessment (TSA) of the power system is a crucial issue with escalating demands and large operational constraints. Real-time TSA allows for deciding and monitoring of the relevant preventive/corrective control actions depending on the dynamic behavior of the system components. To assess this, coherency of generating machines is to be found. After determination of the coherent machines, any corrective or preventive action can be initiated by the system operator to maintain stability of the system during occurrence of any severe contingency. The Transient Severity Index (TSI) introduced in this paper has proven to be an interesting alternative for determining generator coherency. Furthermore, the numerical values of this index are employed to construct a supervised learning-based classifier and the ranking method with the help of system load and generation as input features. This framework employs the support vector machine (SVM) to perform the ranking of the generators based on severity and classify them into vulnerable and nonvulnerable machines. The results are validated on the IEEE 10generator, 39-bus test (New England) system. It is observed that the proposed index and the supervised learning engine give satisfactory results and both are aligned with the published approaches.
\end{abstract}

\section{Introduction}

Modern power network is emerged from the vertically integrated structure to the deregulated unbundled structure. This transformation enables consumers and generators to participate in the competitive business environment. Issues of power quality, reliability, risk, and security become prominent in this environment. High power demand has made an essential criterion to maintain the power system secure under the stressed operating conditions. Transient stability (T/S) of the power system is an important denominator and defined by the CIGRE committee [1] as "the ability of an electric power system, for a given initial operating condition, to regain a state of operating equilibrium after being subjected to a physical disturbance, with most system variables bounded so that practically the entire system remains intact." Stability studies can be subdivided into two categories: the first one is assessment and the second one is control. Ensuring T/S under any unforeseen contingency is a challenge for a system operator. This fact initiated the research in the direction of assessment methods along with preventive control methods for ensuring system stability.

In the past, various methods have been applied by researchers to assess system stability. Stability analysisoriented research is mainly based on the evaluation of the violation of the limit of power system quantities and the behavior of the generator under contingency conditions. Some methods [2-5] proposed for stability assessment in the latest literature were based on risk or probabilistic, pattern recognition, and time domain simulation (TDS) techniques. TSA is directly concerned about the large disturbance. A risk-based methodology was proposed in [2-4]. A probabilistic small-disturbance stability analysis (PSSA) method with uncertainties was proposed by Preece and Milanović [2]. In this approach, the probability density functions (PDFs) were designed for the damping of the critical oscillatory electromechanical modes by modeling the stochastic variation 
of system uncertainties. However, this article did not give any analysis for large disturbances. A linear function-based technique was used in [3] to calculate a risk-based index for TSA. A new technique to identify the severity of the power substation under possible operating scenarios was proposed by Da Silva et al. [4]. The research work presented in [5] was based on the pattern discovery-based fuzzy classification. The pattern identification algorithm was modified by using centroid deviation analysis and predisturbance information of the training data set. This centroid deviation analysis-based technique is very difficult for practical implementation. The Lyapunov stability criteria were given in [6] for differential/algebraic models of power systems which include the effect of generator damping and nonlinear loads. Cheung and Chow [7] investigated the simulation of slow dynamics in two-time-scale power systems. In this work, an approach was proposed to obtain the slow dynamics by projecting the trajectory of the postfault system onto its slow manifold. Direct stability analysis of AC/DC power systems using a structure-preserving energy function (SPEF) was proposed in [8]. Bhat et al. [9] gave a concept of the single machine equivalent (SIME) T/S method combined with Simulink to model T/S phenomena and the Graphical User Interface Design Environment (GUIDE) software of MATLAB. A method based on the network decomposition algorithm was presented in [10] to implement simultaneous unbalances in power system $\mathrm{T} / \mathrm{S}$.

In the current decade, many researchers have proposed stability indices for assessment of stability of large power systems. Transient security indices determined in $[11,12]$ can be used to measure the synchronism state of a generating unit. A small signal stability index was proposed in [13] for power network dynamic assessment by employing TDS. The value of this index was calculated by the systems' eigenvalues using dynamic simulation. Several indices were presented in $[14,15]$ for TSA. A practical and heuristic index based on $\mathrm{T} / \mathrm{S}$ in large power systems was proposed in [14] for fast contingency ranking. A didactic procedure based on simulation for T/S of a multimachine power system was proposed in [16]. Some of the techniques presented in the literature were based on the element T/S, angle stability index, frequency stability index, and transient voltage stability index.

As stated, generator coherency has a considerable application in power system operation and control. The sole concept of coherent groups of generators in a power system is the similar behavior of TDS responses of generators, when they are subjected to a perturbation [17]. This is called coherency. In this consideration, generators that have the similar postdisturbance rotor angle or speed variation characteristics are called coherent and are placed in the similar coherent group. Hence, it is required to monitor and examine the relationship or similarity of rotor angle deviation to find the coherent nature of the power system components. The generators' dynamic response under disturbances can also be recognized by the deviations in phase angles of voltage or/and current phasors of the system.

Several methods have been introduced in the literature for identification of coherent behavior of the generators and classification of them, according to their similar behavior (TDS characteristics). After identification of the coherent groups, control strategies can be applied on them. In order to initiate any preventive control under stressed condition, it is desirable to find the coherency between generators. In general, coherency classification techniques can be divided mainly into two types. The techniques that are placed in the first type are based on model reduction and required computation of the eigenvalues and eigenvectors of the power system [18]. For example, a synchronic modal equivalencing (SME) was proposed [18] for structurepreserving dynamic equivalencing of large power system models. Techniques that are placed in the second type are based on disturbances and use TDS to find coherent groups of generators. For example, several studies have used the rotor trajectory index (RTI) [12], Fourier spectrum [19], or fast Fourier dominant interarea mode [20], principal component analysis [21], independent component analysis [22], hierarchical clustering methods [23, 24], fuzzy c-medoids algorithm [25, 26], wavelet [27], and HilbertHuang transform [28] to find coherent generators. Several techniques proposed in literature cannot always be reliable for all applications, such as system protection schemes or remedial action schemes, dynamic equivalencing, and controlled islanding. This maloperation is found due to the degree of coherency between generators or nongenerating buses. This may change for different disturbances at different power system operating conditions.

Generally, real-time TSA is an approach to find the fast and accurate prediction of the system stability status (either stable or unstable) in real time by considering the future behavior of the generator under the disturbed operating condition. In the literature, various topologies were proposed to forecast the system stability states by using different types of artificial intelligence-based techniques [29-32]. Hashiesh et al. proposed a supervised learning technique for T/S state prediction by the artificial neural network (ANN) [30]. The SVM-based techniques [31] were used for power system contingency classification and ranking in $[29,32]$. The real-time TSA method using the SVM was also proposed in [33]. The prediction of the real-time transient state of the power can be done successfully by the above-discussed methods, but these methods are used only as a classifier. The stability status of the individual generator for any contingency cannot be determined by these methods. For the control action like generator rescheduling, knowledge of the individual generator state (either stable or unstable) is mandatory. To overcome these deficiencies, we propose an SVM-based method to predict the status of the individual generator and overall system by using the coherent characteristics of the generators.

In this paper, the T/S status of the power system is analyzed by simulating the power system under different operating conditions, on different fault locations, and for different fault clearing times. The status of system stability is identified by using a new index, Transient Severity Index (TSI). This index is based on rotor angle deviation of the generators in the postfault state. A supervised learning 
engine based on the LS-SVM is proposed to rank the generators according to the values of TSIs. The potential features for this supervised learning engine are extracted from TDS of the power system under dynamic operating conditions. These potential features are real, reactive power at generating and load buses.

The remaining part of this paper is organized as follows: Section 2 describes power system modeling, Section 3 contains the mathematical framework of the proposed TSI along with the model of the LS-SVM. In Section 4, simulation and results are presented, while Section 5 highlights major conclusions drawn from the work and also presents a solid milieu for future work.

\section{Power System Modeling}

The TDS method is used to solve the differential equations involved in power system stability analysis. TDS of the power system is required for DSA to find whether the system can maintain synchronism during disturbance or not. This decision is taken by monitoring the movement of trajectories of rotor angles during a perturbation period. The swing equation shows the transient behavior of the system. If the trajectories of the rotor angle of either a single generator or a group of generators are found to have continuous increment without limit with reference to remaining machines, then the system is unstable. Another phenomenon is if rotor angles of all working system generators remain bounded within their respective permissible limits, then the system is stable $[17,34]$ :

$$
\begin{aligned}
\frac{d \delta_{j}}{d t}= & \Delta \omega_{j}, \\
\frac{d \Delta \omega_{j}}{d t}= & \frac{1}{M_{j}}\left(P_{m j}-P_{e j}-D_{j} \Delta \omega_{j}\right), \\
P= & G_{j j} E_{j}^{2}+\sum_{k=1, k \neq j}^{n} E_{j} E_{k}\left\{G_{j k} \cos \left(\delta_{j}-\delta_{k}\right)\right. \\
& \left.+B_{j k} \sin \left(\delta_{j}-\delta_{k}\right)\right\},
\end{aligned}
$$

where $G_{j k}+j B_{j k}$ is the transfer admittance between the $j$ th and $k$ th generators, $M_{j}$ is the moment of inertia, $P_{e j}$ is the electrical power output, $D_{j}$ is the damping coefficient, $P_{m j}$ is the mechanical power input, $\Delta \omega_{j}$ is the rotor speed deviation, $\delta_{j}$ is the rotor angle of the $j$ th machine, and $\delta_{k}$ is the rotor angle of the $k$ th machine. The deviation between generator rotor angles with reference to time $t$ can be found by using the set of swing equations (1)-(3):

$$
\delta_{\mathrm{COI}}(t)=\frac{1}{M_{\text {total }}} \sum_{j=1}^{N_{G}} M_{j} \delta_{j}(t) .
$$

In the projectile transient phenomena, the inertial center of the system is taken as the reference frame for the calculations. The machines' rotor angles with respect to center of inertia (COI) [35] are used to detect whether the system is stable or not. For an $N_{G}$ - machine system with the inertia constant $M_{j}$ and rotor angle $\delta_{j}$ of the $j$ th machine, the inertial center $\delta_{\mathrm{COI}}$ is determined as per (4).

\section{Proposed Methodology}

In this work, a numerical integration technique (RungeKutta method) is used to solve the swing equation. The transient security status is determined by monitoring the swing in rotor angle trajectories and deviation in rotor angle with respect to the constraint for transient instability. The constraint is given as follows:

$$
\Delta \delta_{j, \mathrm{COI}}=\left|\delta_{j}-\delta_{\mathrm{COI}}\right| \leq \delta_{\max } \text { for } j=1,2, \ldots, N_{G},
$$

where $\delta_{\max }$ is the maximum allowable value of the relative rotor angle for secure operation. During the case of disturbance, if the relative rotor angle $\Delta \delta_{j, \mathrm{COI}}$ violates $\Delta \delta_{j, \mathrm{COI}}\left(\geq \delta_{\max }\right)$ in a time interval $\left[0, t_{\max }\right]$, then the system is considered as insecure (1), else it is considered as secure (0). For this work, the maximum allowable value of the relative rotor angle for secure operation $\delta_{\max }$ is taken as $120^{\circ}$ [36].

The ranking of the generating machines for every insecure contingency needs to be discovered with less computational burden and time. Therefore, the rotor angle trajectory-based severity index, called the Transient Severity Index (TSI), is proposed in this paper. The TSI is determined from TDS, and the process of determination is shown in Figure 1. The TSI can be used to assess the stability of the power system and to rank the criticality of the generators. Hence, it gives the synchronizing condition for a given hard contingency. In this work, we present a method to rank the machines by employing the TSI. Equation (6) shows the mathematical formulation of the TSI:

$$
\operatorname{TSI}_{j}=1-\frac{K-\Delta \delta_{j, \mathrm{COI}}(\tau)}{K+\Delta \delta_{j, \mathrm{COI}}(\tau)}
$$

where $\Delta \delta_{j, \mathrm{COI}}$ is the value of rotor angle deviation in degrees, $K$ is the trajectory constant, and $\tau$ is the time at which $\Delta \delta_{j, \mathrm{COI}}$ is measured. $\Delta \delta_{j, \mathrm{COI}}$ for all the generators $\left(j=1,2, \ldots, N_{G}\right)$ is noted after 48 cycles of fault clearing time (FCT). A classification boundary is shown in Table 1 . The numerical value of the TSI is an indicator of the unstable or stable system state of the power system.

$$
\text { System state }= \begin{cases}\text { unstable } & \text { if } \mathrm{TSI} \geq 1 \\ \text { stable } & \text { if } \mathrm{TSI}<1\end{cases}
$$

The generators can be divided into three groups: the first is highly critical (most advanced) generators (CGs), the second is advanced generators, and the third is noncritical (least advanced) generators (NGs). Ranking of generators can be found by their corresponding generator TSI value. Furthermore, this ranking can be used in the process of preventive and corrective control action.

3.1. Sensitivity Analysis of $K$. In this section, sensitivity analysis of the trajectory constant $(K)$ is presented. On the basis of the values of $K$, the TSI can take any three values as per (8)-(10). 


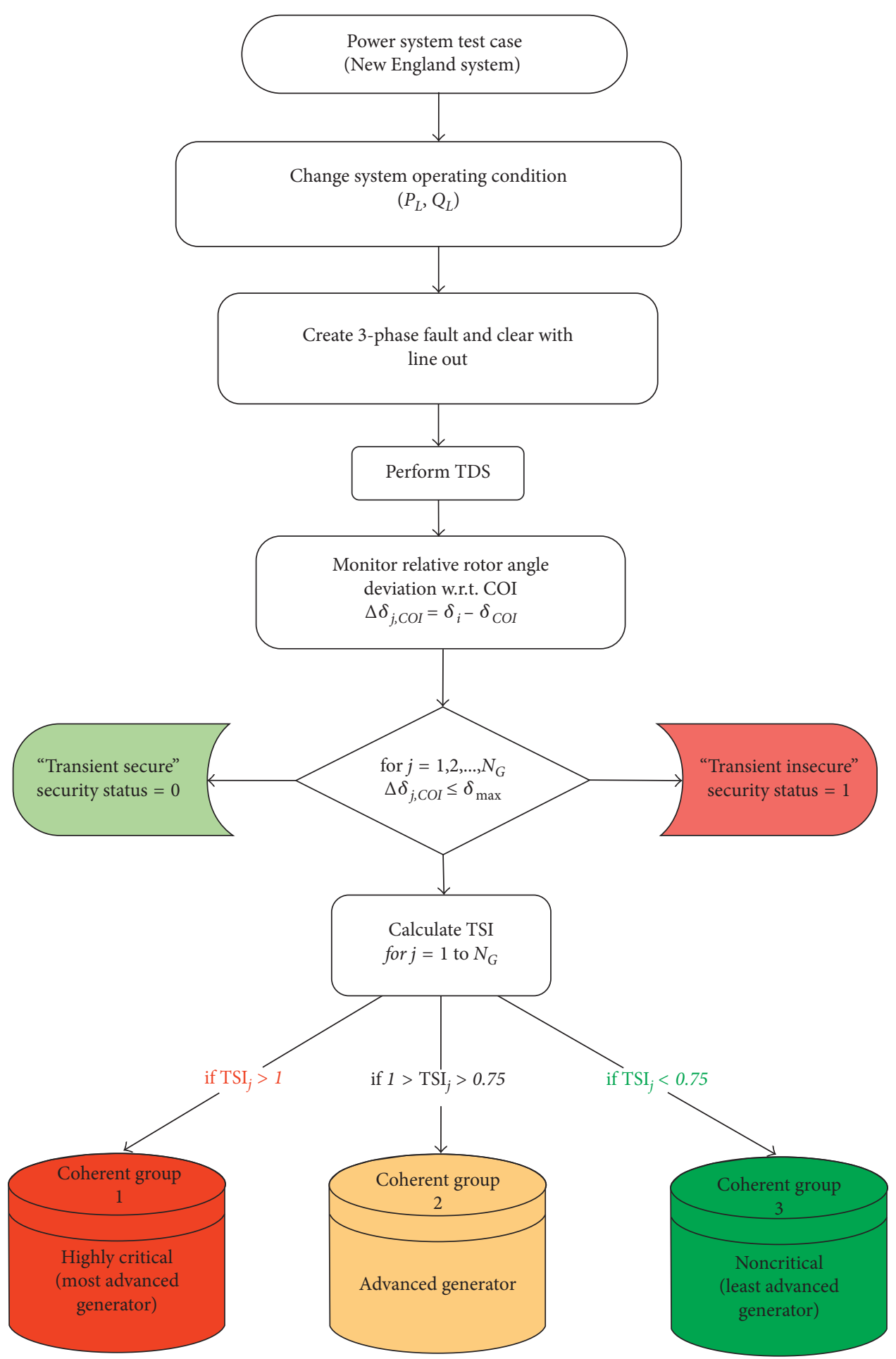

FIgURE 1: Flow chart of proposed assessment and identification scheme for transient stability.

TABle 1: Classification of coherent groups.

\begin{tabular}{lccc}
\hline Range of the TSI & Coherent group & Criticality of the generator & Preventive action (rescheduling) \\
\hline TSI $1 \geq 1$ & 1 & Highly critical (most advanced generator) & $P_{G}$ decreases \\
$1 \geq$ TSI $\geq 0.75$ & 2 & Advanced generator & - \\
$0.75>$ TSI & 3 & Noncritical (least advanced generator) & $P_{G}$ increases \\
\hline
\end{tabular}




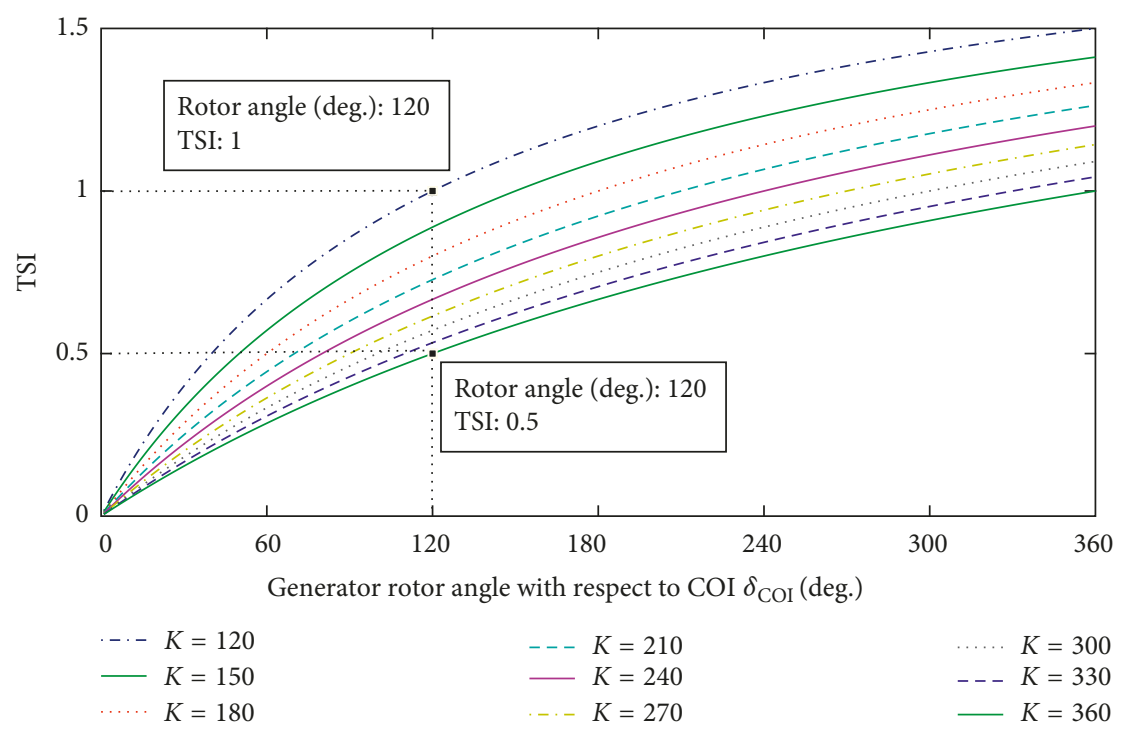

FIgURE 2: TSI value with respect to rotor angle deviation for different values of $K$.

Case $1: K<\Delta \delta_{j, \mathrm{COI}}$

$$
\mathrm{TSI}=\left[1+\frac{\Delta \delta_{j, \mathrm{COI}}(\tau)-K}{\Delta \delta_{j, \mathrm{COI}}(\tau)+K}\right]<1 .
$$

Case $2: K>\Delta \delta_{j, \mathrm{COI}}$

$$
\mathrm{TSI}=\left[1-\frac{K-\Delta \delta_{j, \mathrm{COI}}(\tau)}{K+\Delta \delta_{j, \mathrm{COI}}(\tau)}\right]>1 .
$$

Case 3: $K=\Delta \delta_{j, \mathrm{COI}}$

$$
\mathrm{TSI}=1 .
$$

The variation of $\Delta \delta_{j, \text { COI }}$ and TSI with respect to $K$ is observed, and the results of this analysis are exhibited in Figure 2. It shows TSI trajectories for nine different values of $K$. $K$ varies from $120^{\circ}$ to $360^{\circ}$ in the step of $30^{\circ}$. By inspecting the variation of the TSI values with respect to $\Delta \delta_{j, \mathrm{COI}}$ for the constant value of $K$, that higher value of $K$ reduces the span of the index. As the value of $K$ decreases, the span of the index increases. It is a basic characteristic to design a scalar index. Hence, the trajectory at $K=120^{\circ}$ (i.e., $\delta_{\max }$ ) exhibits the largest span to classification and is used for further analysis.

3.2. Design of the Supervised Learning Engine. The values of the rotor angle are viewed as a multidimensional vector (a list of index values), and a classification boundary (hyperplane) is required to classify the generators in the abovementioned three coherent groups. Design of the SVM has two aspects: the first is to reduce the number of features by any feature selection method and the second is to find the largest margin (hyperplane) between both the states of the system (stable and unstable). For handling these aspects, particle swarm optimization (PSO) [37-39] is employed in this work.

The TSI can be used to assess the stability of the power system and rank the generator. A supervised learning engine based on the LS-SVM is proposed to predict the coherent
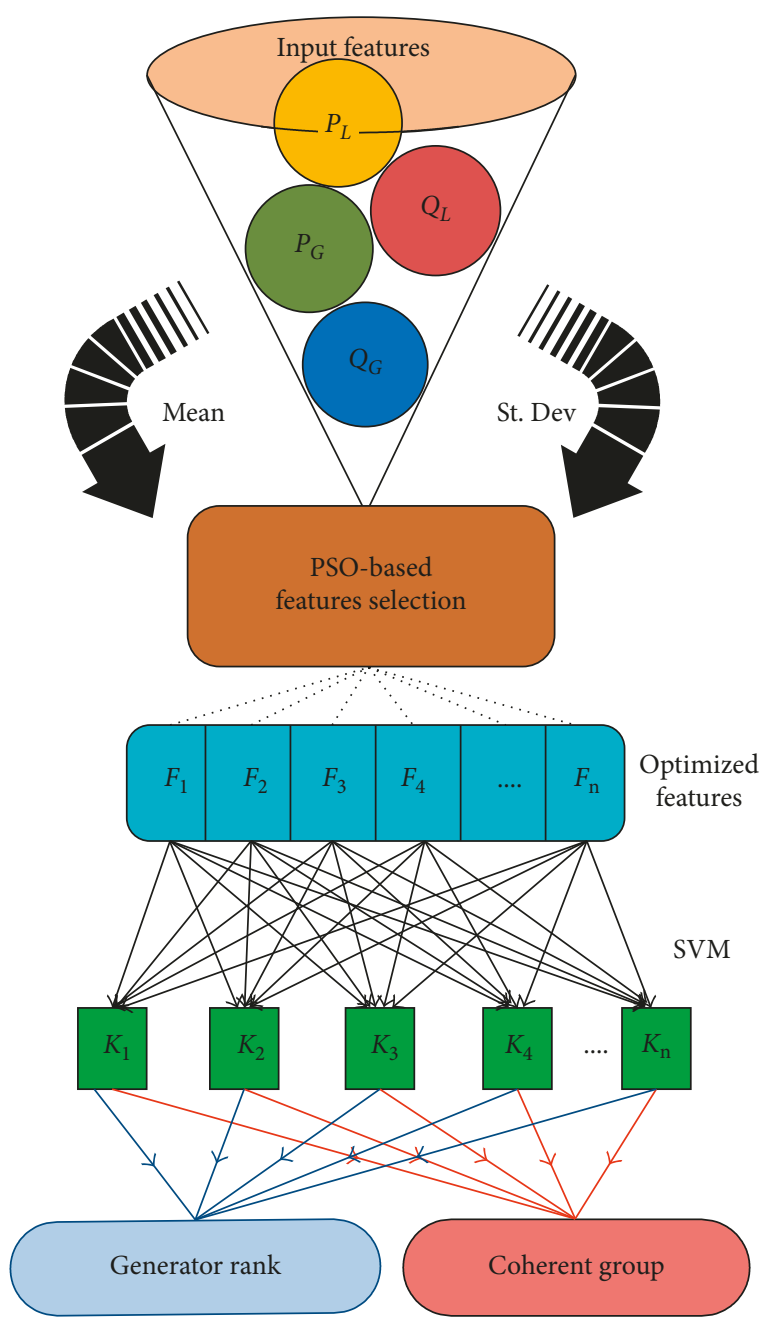

FIGURE 3: Least-square support vector machine network. 


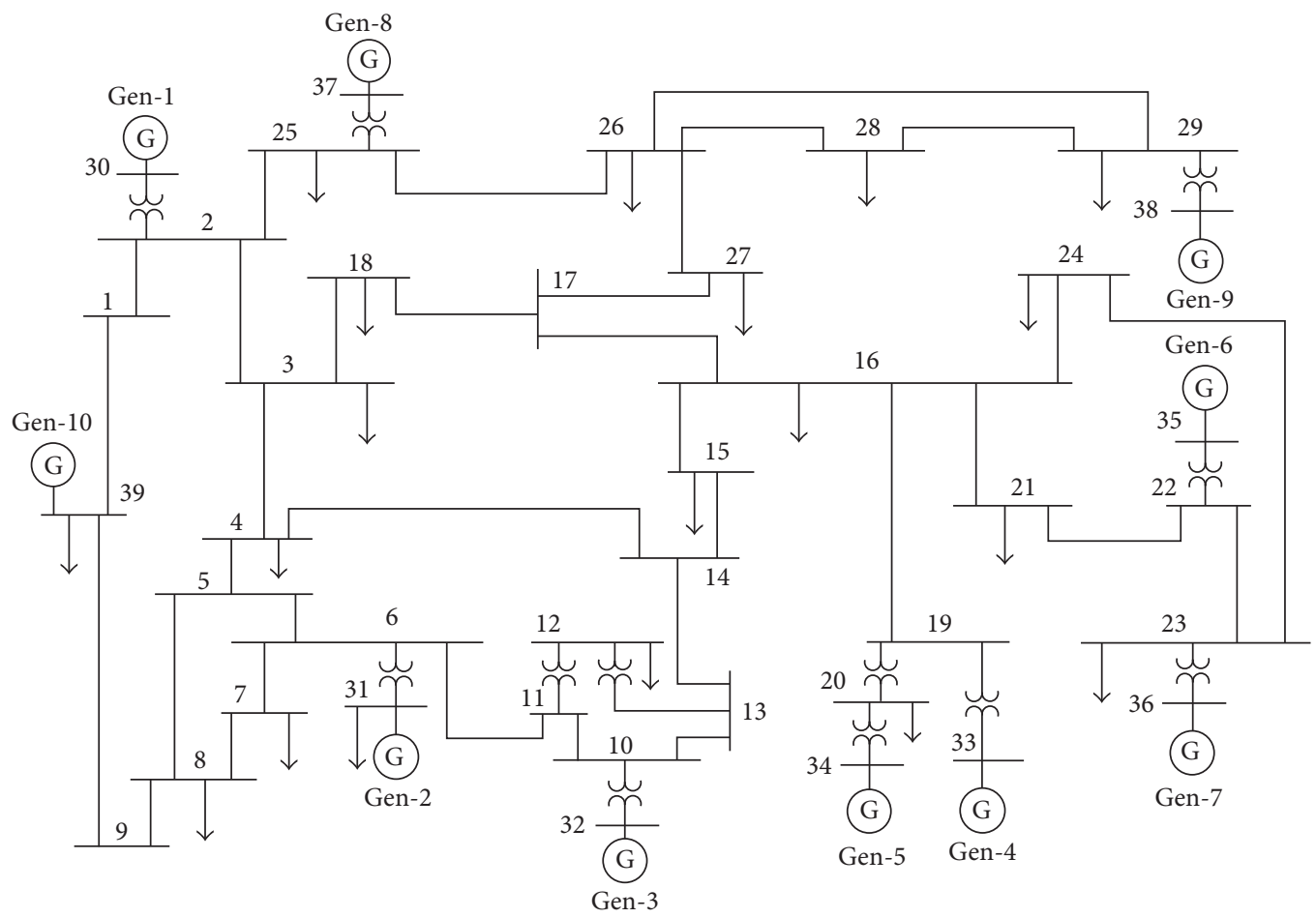

Figure 4: IEEE New England power system.

group and rank the generators according to the values of the TSI as depicted in Figure 3. In LS-SVMs, the input data are mapped in a linear form with the high-dimensional feature space with the help of kernel functions. To solve the classification problem, least-square loss function is used in this paper.

The input features vector for this engine consisting of real and reactive power at generator buses $\left(P_{G}\right.$ and $\left.Q_{G}\right)$ and load buses $\left(P_{L}\right.$ and $\left.Q_{L}\right)$ is defined by the following equation:

$$
\text { Input vector }\left[x_{n}\right]=\left[P_{G j}, Q_{G j}, P_{L i}, Q_{L i}\right] \text {, }
$$

where $j=1,2, \ldots, N_{G}$ and $i=1,2, \ldots, N_{L}$, in which $N_{G}$ and $N_{L}$ represent the total no. of generator buses and load buses, respectively.

Feature selection aims to find the most important information from a given set of features. As this task can be seen as an optimization problem, the combinatorial growth of the possible solutions may be inviable for an exhaustive search. In this paper, PSO is used for feature selection and SVM is used for maximum margin classifier design [40]. A mean of standard deviation-based objective function is optimized by using PSO. In this approach, PSO creates different data packets with random feature selection and obtains the standard deviation of each data packet and maximizes the mean of the standard deviation of different data packets. The final feature vector is that which has the maximum mean or large Euclidean distance of features. The vector of potential input features for training of the SVM can be found from the following equation:

$$
\begin{aligned}
F= & {\left[P_{L 3}, P_{L 5}, Q_{L 2}, Q_{L 3}, Q_{L 10}, Q_{L 13}, Q_{L 15}, Q_{L 18}, Q_{L 20},\right.} \\
& \left.P_{G 2}, P_{G 3}, P_{G 6}, P_{G 9}, Q_{G 2}, Q_{G 4}, Q_{G 5}, Q_{G 7}\right] .
\end{aligned}
$$

The target for the SVM output is the generator severity rank and coherent group of all the generators at a given time instant, which is defined as target vector as shown in the following equation:

$[Y]=$ [generator severity rank, coherent group].

Authors simulated 1,000 different cases; out of these, $85 \%$ are used for training and validation and the remaining $15 \%$ are used for testing purpose.

\section{Simulation Results}

The proposed strategy is tested on the global standard benchmark IEEE 10-generator and 39-bus (New England) power network as shown in Figure 4. The system has 46 transmission lines, 39 buses, 10 generators, and 12 transformers. Bus 39 is considered as the slack bus during simulations. All the TDS works are carried out using MATLAB [41], MATPOWER [42], and PSAT Version 2.1.10 [43] with Intel Core $^{\mathrm{TM}}$ i3-3110M platform with $2.40 \mathrm{GHz}$ and 6 GB RAM. The symmetrical fault with ground is initiated at random time from $1.1 \mathrm{~s}$ to $1.2 \mathrm{~s}$ and cleared at $1.3 \mathrm{~s}$ with different random perturbations of the real and reactive loads on all the buses and real and reactive power generation at the generator buses. System total load is varied from $95 \%$ to $110 \%$ of the base case total load. Fault is applied on all types of buses. To demonstrate the effectiveness of the proposed method, six typical severe 
TABLe 2: Application of credible contingencies.

\begin{tabular}{lccccc}
\hline Contingency & Type of fault & Location of fault & Tripped line & Fault duration (freq. 60 Hz) & System load (\% of base case) \\
\hline Case I & 3-phase fault & Bus 13 & Line 13-14 & 10.37 cycles & $97.50 \%$ \\
Case II & 3-phase fault & Bus 7 & Line 6-7 & 9.0 cycles & $110.00 \%$ \\
Case III & 3-phase fault & Bus 10 & Line 10-11 & 10.0 cycles & $95.00 \%$ \\
Case IV & 3-phase fault & Bus 14 & Line 14-15 & 6.5 cycles & $105.00 \%$ \\
Case V & 3-phase fault & Bus 26 & Line 26-27 & 8.3 cycles & Base case \\
Case VI & 3-phase fault & Gen. 4 (bus 34) & - & 11.69 cycles & $105.00 \%$ \\
\hline
\end{tabular}

TABLE 3: Classification and ranking obtained by the supervised learning engine (LS-SVM).

\begin{tabular}{|c|c|c|c|c|c|c|c|c|c|c|}
\hline & & G1 & G2 & G3 & G4 & G5 & G6 & G7 & G8 & G9 \\
\hline \multirow{5}{*}{ Case I } & RTI [12] & 0.333 & 0.866 & 1.007 & 0.477 & 0.485 & 0.462 & 0.477 & 0.414 & 0.476 \\
\hline & TSI & 0.749 & 1.393 & 1.505 & 0.969 & 0.98 & 0.948 & 0.969 & 0.879 & 0.968 \\
\hline & Rank & 9 & 2 & 1 & 4 & 3 & 7 & 5 & 8 & 6 \\
\hline & Coherency group & 3 & 1 & 1 & 2 & 2 & 2 & 2 & 2 & 2 \\
\hline & Stability status & 0 & 1 & 1 & 0 & 0 & 0 & 0 & 0 & 0 \\
\hline \multirow{5}{*}{ Case II } & RTI [12] & 0.41 & 0.494 & 0.511 & 0.509 & 0.539 & 0.477 & 0.485 & 0.366 & 0.405 \\
\hline & TSI & 0.873 & 0.815 & 0.903 & 1.012 & 1.05 & 0.967 & 0.978 & 0.802 & 0.864 \\
\hline & Rank & 6 & 8 & 5 & 2 & 1 & 4 & 3 & 9 & 7 \\
\hline & Coherency group & 2 & 2 & 2 & 1 & 1 & 2 & 2 & 2 & 2 \\
\hline & Stability status & 0 & 0 & 0 & 1 & 1 & 0 & 0 & 0 & 0 \\
\hline \multirow{5}{*}{ Case III } & RTI [12] & 0.402 & 0.499 & 0.614 & 0.466 & 0.488 & 0.482 & 0.486 & 0.395 & 0.402 \\
\hline & TSI & 0.86 & 0.969 & 1.096 & 0.954 & 0.984 & 0.976 & 0.981 & 0.85 & 0.86 \\
\hline & Rank & 7 & 5 & 1 & 6 & 2 & 4 & 3 & 9 & 8 \\
\hline & Coherency group & 2 & 2 & 1 & 2 & 2 & 2 & 2 & 2 & 2 \\
\hline & Stability status & 0 & 0 & 1 & 0 & 0 & 0 & 0 & 0 & 0 \\
\hline \multirow{5}{*}{ Case IV } & RTI [12] & 0.396 & 0.341 & 0.365 & 0.518 & 0.529 & 0.466 & 0.469 & 0.334 & 0.414 \\
\hline & TSI & 0.851 & 0.537 & 0.594 & 1.024 & 1.033 & 0.943 & 0.956 & 0.744 & 0.872 \\
\hline & Rank & 6 & 9 & 8 & 2 & 1 & 4 & 3 & 7 & 5 \\
\hline & Coherency group & 2 & 3 & 3 & 1 & 1 & 2 & 2 & 3 & 2 \\
\hline & Stability status & 0 & 0 & 0 & 1 & 1 & 0 & 0 & 0 & 0 \\
\hline \multirow{5}{*}{ Case V } & RTI [12] & 0.273 & 0.366 & 0.392 & 0.39 & 0.418 & 0.393 & 0.395 & 0.418 & 1.131 \\
\hline & TSI & 0.604 & 0.771 & 0.813 & 0.81 & 0.852 & 0.812 & 0.826 & 0.712 & 1.592 \\
\hline & Rank & 9 & 7 & 4 & 6 & 2 & 5 & 3 & 8 & 1 \\
\hline & Coherency group & 3 & 2 & 2 & 2 & 2 & 2 & 2 & 3 & 1 \\
\hline & Stability status & 0 & 0 & 0 & 0 & 0 & 0 & 0 & 0 & 1 \\
\hline \multirow{5}{*}{ Case VI } & RTI [12] & 0.244 & 0.346 & 0.362 & 0.461 & 1.324 & 0.373 & 0.384 & 0.275 & 0.298 \\
\hline & TSI & 0.365 & 0.734 & 0.752 & 0.625 & 1.709 & 0.697 & 0.682 & 0.562 & 0.618 \\
\hline & Rank & 9 & 3 & 2 & 6 & 1 & 4 & 5 & 8 & 7 \\
\hline & Coherency group & 3 & 3 & 2 & 3 & 1 & 3 & 3 & 3 & 3 \\
\hline & Stability status & 0 & 0 & 0 & 0 & 1 & 0 & 0 & 0 & 0 \\
\hline
\end{tabular}

contingencies have been taken into consideration as described in Table 2 for studies. The proposed procedure is utilized to identify coherency information for generation rescheduling to initiate preventive control. The experimental procedure and its evaluation methodology are as follows:

Step 1: For any insecure contingency, after the observation of rotor angle trajectories, three coherent groups are made to classify all the generators according to the value of the TSI as in Table 1.

Step 2: All the critical generators (most advanced generators), also considered in a group of critical machines, form the generation decreasing group.

Step 3: All noncritical machines or machines of the least advanced generator group form the generation increasing group.
Step 4: After the coherency and stability assessment, initiate the preventive control as suggested by the proposed method.

Table 3 shows that the results (rank and coherent group) obtained from the LS-SVM are aligned with RTI [12]. For the justification of these results, TDS is also performed, and the results of six cases are presented below.

Case I is shown for $97.5 \%$ random load variation, and three-phase fault at bus 13 is initiated at $1.1272 \mathrm{~s}$ and cleared at $1.3 \mathrm{~s}$ by opening the breakers to isolate the line 13-14. The state of $\mathrm{T} / \mathrm{S}$ for all generators is determined by observing rotor angle swings in the time interval. As per Table 3, TSI value of the generators G1 and G2 is violate stability criteria (i.e., TSI > 1), so these generators are considered as unstable generators. Figure 5 shows the relative rotor angle of all the generators with respect to COI. The rotor swing of the 


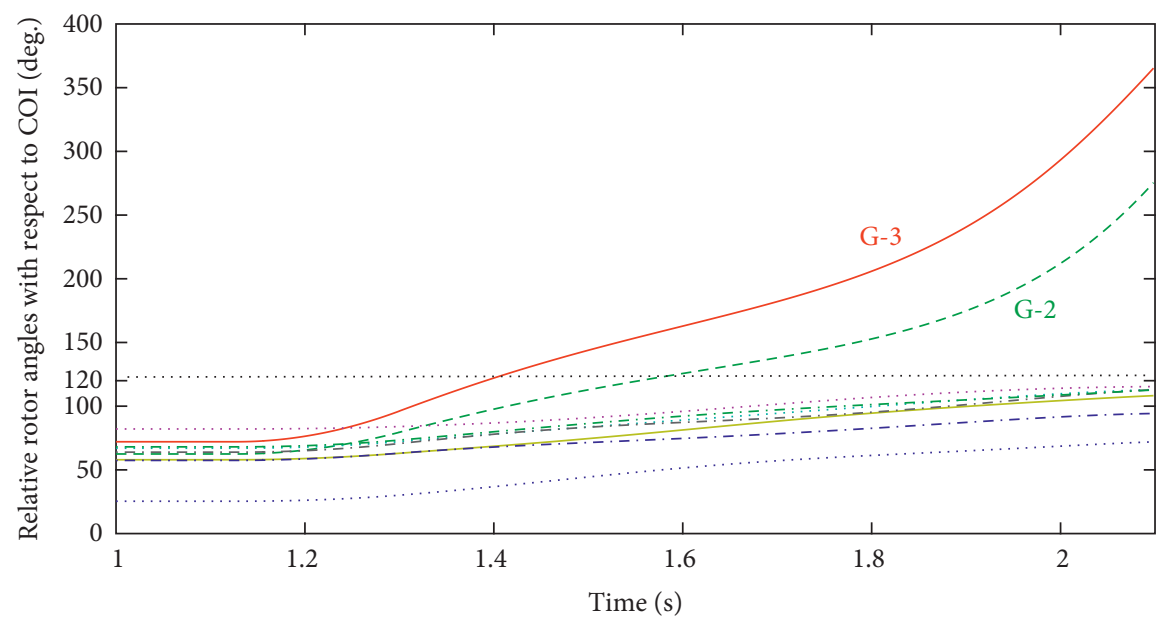

FIgURE 5: Postfault TDS response of rotor angle deviation of the generator for case I.

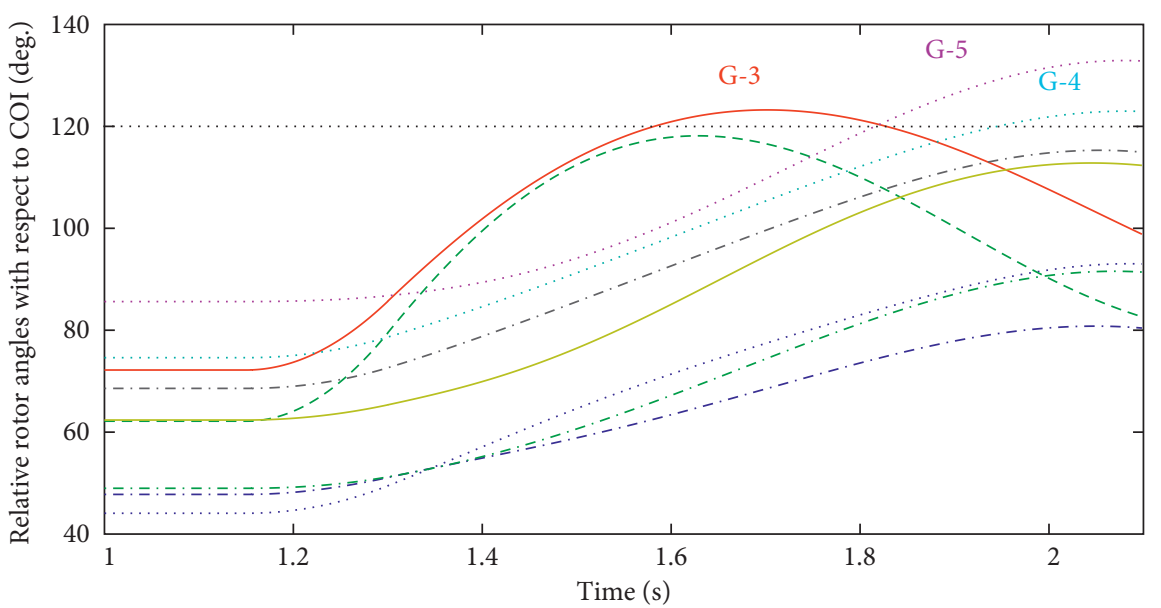

FIgURE 6: Postfault TDS response of rotor angle deviation of the generator for case II.

generators G2 (at bus 31) and G3 (at bus 32) violates the threshold value, causing them to lose synchronism. Both the generators are going out of step with respect to the other generators, making the whole system transiently unstable.

Case II is shown for $110 \%$ random load variation, and three-phase fault at bus 7 is initiated at $1.1498 \mathrm{~s}$ and cleared at $1.3 \mathrm{~s}$ by opening the breakers to isolate the line 6-7. As per Table 3, TSI values of the generators G4 and G5 violate stability criteria (i.e., TSI $>1$ ), so these generators are considered as unstable generators. Classification results for G3 obtained from the RTI and TSI are conflicting. Figure 6 shows the relative rotor angle of all the generators with respect to COI. The rotor swing of the generators G4 (at bus 33) and G5 (at bus 34) rises above the threshold value, causing them to lose synchronism. Both the generators are going out of step with respect to the other generators, making the whole system transiently unstable. However, the generator G3 (at bus 32) is also going out of the stability equilibrium, but after FCT, it regains the stable state after a few cycles. TDS shown in Figure 6 indicates that this generator exhibits self-healing characteristics and does not pose any threat to system stability.
Case III is shown for 95\% random load variation, and three-phase fault at bus 10 is initiated at $1.1468 \mathrm{~s}$ and cleared at $1.3 \mathrm{~s}$ by opening the breakers to isolate the line 10-11. As per Table 3, TSI value of the generator G3 is more then stability criteria (i.e., TSI $>1$ ), so this generator is considered as an unstable generator. Figure 7 shows the relative rotor angle of all the generators with respect to COI. The rotor swing of the generator G3 (at bus 32) violates the threshold value, causing it to lose synchronism. This generator is going out of step with the rest of the generators, making the whole system transiently unstable. However, the generator G2 (at bus 31) is also going out of step, but after FCT, it regains its stable state after a few cycles. TDS shown in Figure 7 indicates that this generator exhibits self-healing characteristics and does not pose any threat to system stability.

Case IV is shown for $105 \%$ random load variation, and three-phase fault at bus 14 is initiated at $1.1917 \mathrm{~s}$ and cleared at $1.3 \mathrm{~s}$ by opening the breakers to isolate the line 14-15. The state of T/S for all generators is determined by observing rotor angle swings in the time interval. As per Table 3, TSI values of the generators G4 and G5 are more 


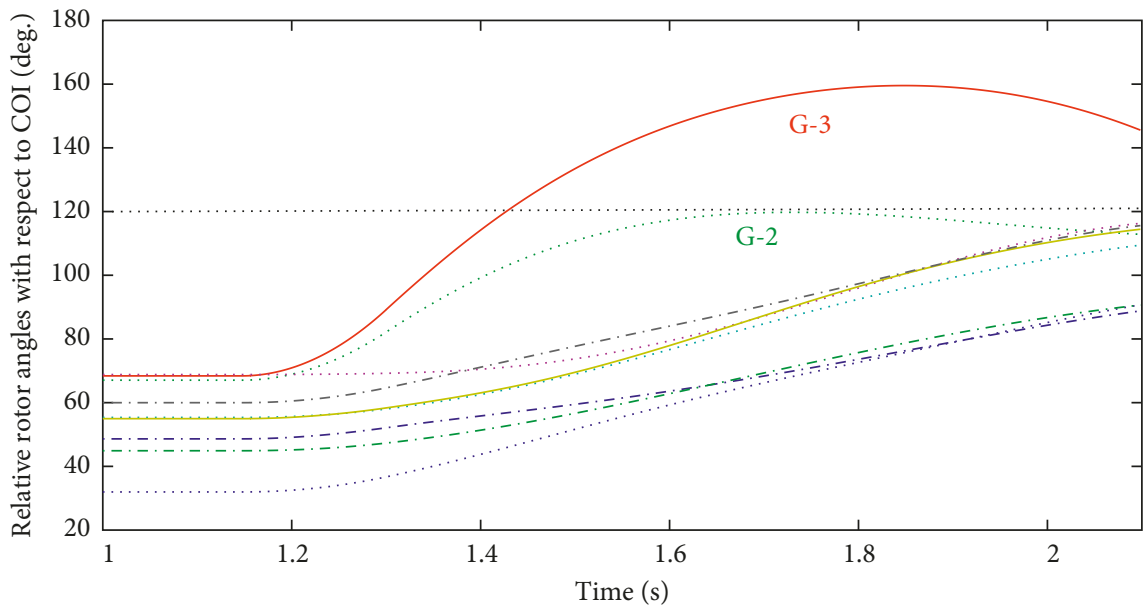

Figure 7: Postfault TDS response of rotor angle deviation of the generator for case III.

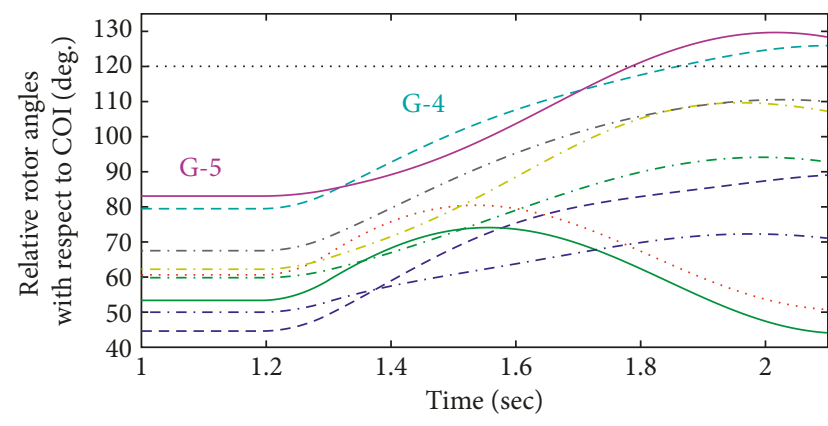

Figure 8: Postfault TDS response of rotor angle deviation of the generator for case IV.

than stability criteria (i.e., TSI $>1$ ), so these generators are considered as unstable generators. Figure 8 shows the relative rotor angle of all the generators with respect to COI. The rotor swing of the generators G4 (at bus 33) and G5 (at bus 34) violates the threshold value, causing them to lose synchronism. Both the generators are going out of step with respect to the other generators, making the whole system transiently unstable.

Case V is shown for the base case load, and three-phase fault at bus 26 is initiated at $1.1610 \mathrm{~s}$ and cleared at $1.3 \mathrm{~s}$ by opening the breakers to isolate the line 26-27. As per Table 3, TSI value of the generator G9 is more than stability criteria (i.e., TSI $>1$ ), so this generator is considered as an unstable generator. Figure 9 shows the relative rotor angle of all the generators with respect to COI. The rotor swing of the generator G9 (at bus 38) violates the threshold value, causing it to lose synchronism. This generator is going out of step with the other generators, making the whole system transiently unstable.

Case VI is shown for $105 \%$ random load variation, and three-phase fault at generator no. 5 , at bus 34 is initiated at $1.1052 \mathrm{~s}$ and cleared at $1.3 \mathrm{~s}$. The state of T/S for all generators is determined by observing rotor angle swings in the time interval. As per Table 3, TSI value of the generator G5 is more then stability criteria (i.e., TSI $>1$ ), so

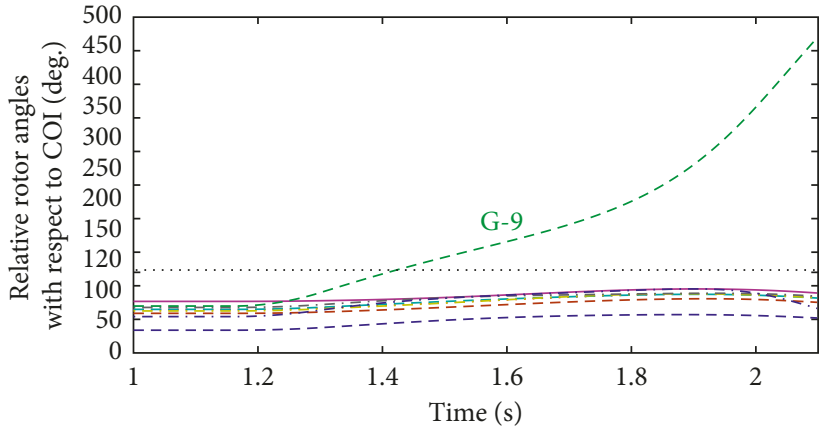

FIgURE 9: Postfault TDS response of rotor angle deviation of the generator for case $\mathrm{V}$.

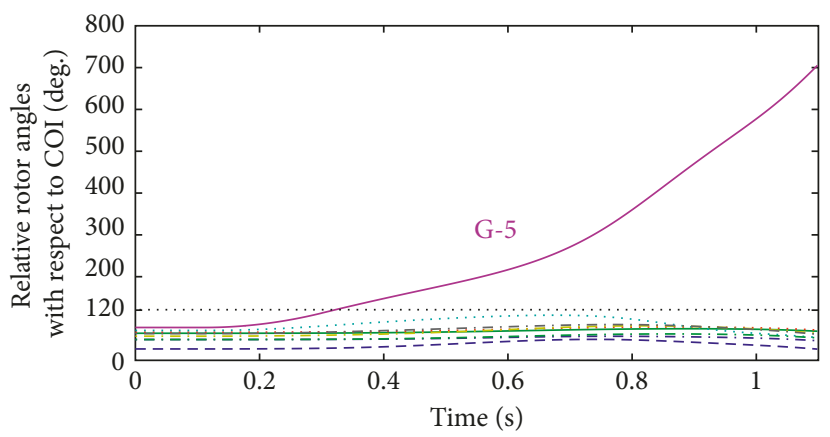

FIgure 10: Postfault TDS response of rotor angle deviation of the generator for case VI.

this generator is considered as an unstable generator. Figure 10 shows the relative rotor angle of all the generators with respect to COI. The rotor swing of the generator G5 (at bus 34) violates the threshold value, causing it to lose synchronism. This generator is going out of step with the rest of the generators, making the whole system transiently unstable. To make the system stable for this case, the proposed method suggests rescheduling of the generating units. 


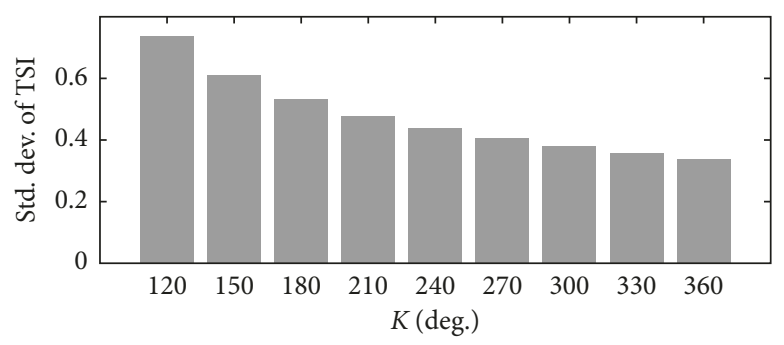

(a)

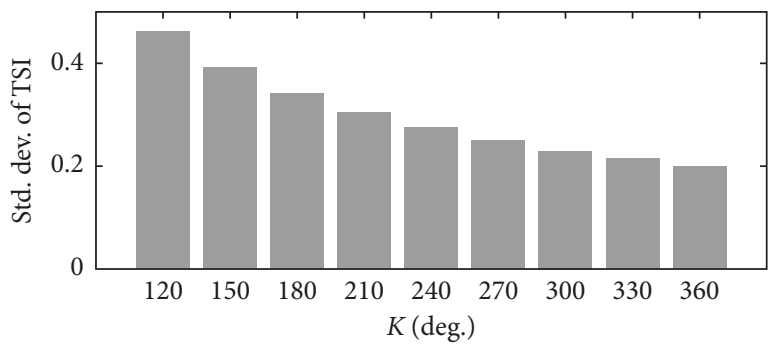

(c)

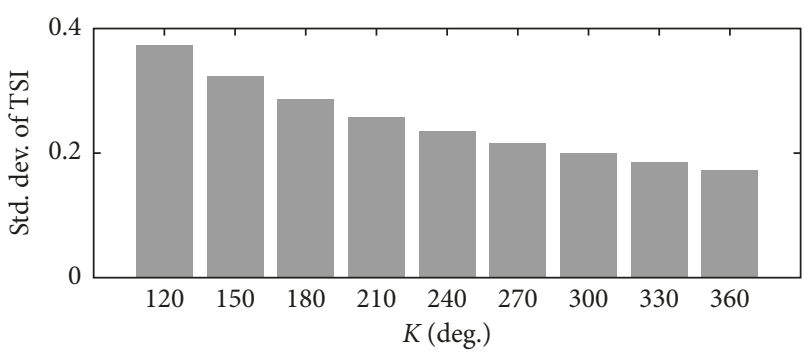

(b)

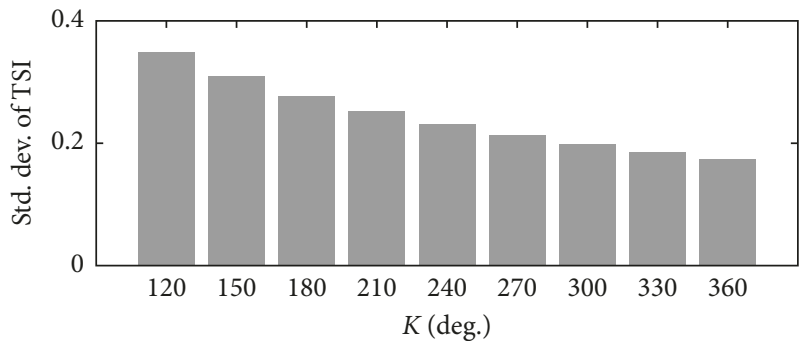

(d)

FIgURE 11: Standard deviation of TSI values at different values of $K$ : (a) case I, (b) case II, (c) case III, and (d) case IV.

Results shown in Table 3 are validated through nonlinear TDS as shown in Figures 5-10. It is observed that the results of TDS and the supervised learning architecture are aligned with each other. This comparison establishes the efficacy of the proposed approach.

Figure 11 shows the value of standard deviation for different values of the trajectory constant. It has been observed that the standard deviation is highest for $K=120^{\circ}$, which indicates that the Euclidean distance of the samples is higher for $K=120^{\circ}$ as compared to the others. Hence, these cases are easily separable by a linear discriminant function.

\section{Conclusion}

Power system stability is a matter of concern in the present deregulated scenario. Fast and accurate stability assessment methods are more meaningful in the deregulated and dynamic framework of the power system. The problem of stability assessment has been addressed in this paper. Following are the major conclusions of this work:

(i) The index TSI has been proposed in this paper. This index can serve two objectives: the first is to classify the generators in coherent groups and the second is to rank them according to the severity level.

(ii) A supervised learning engine has been presented. This engine employs the LS-SVM method to rank and classify the generators. A swarm intelligencebased well-known technique PSO has been applied to perform feature selection and SVM to design maximum margin classification. It has been observed that the results of the proposed supervised engine are aligned with the numerical values of TSIs obtained using other approaches. (iii) The results of this supervised engine have been validated through nonlinear TDS. It has been observed that the results of the proposed index and supervised engine are aligned with each other. Furthermore, the TDS results verify the effectiveness of this approach.

The application of different feature selection methods and different topologies of neural architectures lies in the scope of the future work.

\section{Conflicts of Interest}

The authors declare that they have no conflicts of interest.

\section{Acknowledgments}

The authors acknowledge the support and encouragement provided by the authorities of the Malaviya National Institute of Technology, Jaipur, and Swami Keshvanand Institute of Technology, Jaipur.

\section{References}

[1] P. Kundur, J. Paserba, V. Ajjarapu et al., "Definition and classification of power system stability IEEE/CIGRE joint task force on stability terms and definitions," IEEE Transactions on Power Systems, vol. 19, no. 3, pp. 1387-1401, 2004.

[2] R. Preece and J. V. Milanović, "Risk-based small-disturbance security assessment of power systems," IEEE Transactions on Power Delivery, vol. 30, no. 2, pp. 590-598, 2015.

[3] A. Dissanayaka, U. D. Annakkage, B. Jayasekara, and B. Bagen, "Risk-based dynamic security assessment," IEEE Transactions on Power Systems, vol. 26, no. 3, pp. 1302-1308, 2011.

[4] A. M. L. Da Silva, A. Violin, C. Ferreira, and Z. S. Machado, "Probabilistic evaluation of substation criticality based on 
static and dynamic system performances," IEEE Transactions on Power Systems, vol. 29, no. 3, pp. 1410-1418, 2014.

[5] F. Luo, Z. Dong, G. Chen et al., "Advanced pattern discoverybased fuzzy classification method for power system dynamic security assessment," IEEE Transactions on Industrial Informatics, vol. 11, no. 2, pp. 416-426, 2015.

[6] D. J. Hill, I. A. Hiskens, and I. M. Y. Mareels, "Stability theory of differential/algebraic models of power systems," Sadhana, vol. 18, no. 5, pp. 731-747, 1993.

[7] K. W. Cheung and J. H. Chow, "Slow dynamics simulation of power systems," Sadhana, vol. 18, no. 5, pp. 749-760, 1993.

[8] K. Padiyar and H. Sastry, "A structure-preserving energy function for stability analysis of AC/DC systems," Sadhana, vol. 18, no. 5, pp. 787-799, 1993.

[9] S. Bhat, M. Glavic, M. Pavella, T. Bhatti, and D. Kothari, "A transient stability tool combining the SIME method with MATLAB and SIMULINK," International Journal of Electrical Engineering Education, vol. 43, no. 2, pp. 119-133, 2006.

[10] A. Koochaki and S. M. Kouhsari, "Simulation of simultaneous unbalances in power system transient stability analysis," Simulation, vol. 87, no. 11, pp. 976-988, 2011.

[11] A. M. Haidar, M. Mustafa, F. A. Ibrahim, and I. A. Ahmed, "Transient stability evaluation of electrical power system using generalized regression neural networks," Applied Soft Computing, vol. 11, no. 4, pp. 3558-3570, 2011.

[12] K. Verma and K. Niazi, "A coherency based generator rescheduling for preventive control of transient stability in power systems," International Journal of Electrical Power \& Energy Systems, vol. 45, no. 1, pp. 10-18, 2013.

[13] F. R. S. Sevilla and L. Vanfretti, "A small-signal stability index for power system dynamic impact assessment using timedomain simulations," in Proceedings of the 2014 IEEE PES General Meeting-Conference \& Exposition, pp. 1-5, IEEE, National Harbor, MD, USA, July 2014.

[14] S. Grillo, S. Massucco, A. Pitto, and F. Silvestro, "Indices for fast contingency ranking in large electric power systems," in Proceedings of the Melecon 2010-2010 15th IEEE Mediterranean Electrotechnical Conference, pp. 660-666, IEEE, Valletta, Malta, April 2010.

[15] Z. Ming, D. Shi, X. Song, and T. Kuo, "Indices system and methods for power grid security assessment based on dynamic fuzzy," in Proceedings of the 2010 International Conference on Electrical and Control Engineering (ICECE), pp. 3830-3833, IEEE, Wuhan, China, June 2010.

[16] S. Ekinci, H. Lale Zeynelgil, and A. Demiroren, "A didactic procedure for transient stability simulation of a multimachine power system utilizing SIMULINK," International Journal of Electrical Engineering Education, vol. 53, no. 1, pp. 54-71, 2016.

[17] P. Kundur, N. J. Balu, and M. G. Lauby, Power System Stability and Control, Vol. 7, McGraw-Hill, Inc., New York, NY, USA, 1994.

[18] G. N. Ramaswamy, C. Evrard, G. C. Verghese, O. Fillatre, and B. C. Lesieutre, "Extensions, simplifications, and tests of synchronic modal equivalencing (SME)," IEEE Transactions on Power Systems, vol. 12, no. 2, pp. 896-905, 1997.

[19] X. Lei, D. Povh, and O. Ruhle, "Industrial approaches for dynamic equivalents of large power systems," in Proceedings of the 2002 IEEE Power Engineering Society Winter Meeting, vol. 2, pp. 1036-1042, IEEE, New York, NY, USA, January 2002.

[20] M. Jonsson, M. Begovic, and J. Daalder, "A new method suitable for real-time generator coherency determination," IEEE Transactions on Power Systems, vol. 19, no. 3, pp. 1473-1482, 2004.
[21] K. K. Anaparthi, B. Chaudhuri, N. F. Thornhill, and B. C. Pal, "Coherency identification in power systems through principal component analysis," IEEE Transactions on Power Systems, vol. 20, no. 3, pp. 1658-1660, 2005.

[22] M. Ariff and B. C. Pal, "Coherency identification in interconnected power system-an independent component analysis approach," IEEE Transactions on Power Systems, vol. 28, no. 2, pp. 1747-1755, 2013.

[23] H. A. Alsafih and R. Dunn, "Determination of coherent clusters in a multi-machine power system based on wide-area signal measurements," in Proceedings of the 2010 IEEE Power and Energy Society General Meeting, pp. 1-8, IEEE, Minneapolis, MN, USA, July 2010.

[24] M. Davodi, H. Modares, E. Reihani, M. Davodi, and A. Sarikhani, "Coherency approach by hybrid PSO, K-means clustering method in power system," in Proceedings of the 2008 IEEE 2nd International Power and Energy Conference, (PECon 2008), pp. 1203-1207, IEEE, Johor Bahru, Malaysia, December 2008.

[25] I. Kamwa, A. K. Pradhan, and G. Joós, "Automatic segmentation of large power systems into fuzzy coherent areas for dynamic vulnerability assessment," IEEE Transactions on Power Systems, vol. 22, no. 4, pp. 1974-1985, 2007.

[26] I. Kamwa, A. K. Pradhan, G. Joos, and S. R. Samantaray, "Fuzzy partitioning of a real power system for dynamic vulnerability assessment," IEEE Transactions on Power Systems, vol. 24, no. 3, pp. 1356-1365, 2009.

[27] S. Avdakovic, E. Becirovic, A. Nuhanovic, and M. Kusljugic, "Generator coherency using the wavelet phase difference approach," IEEE Transactions on Power Systems, vol. 29, no. 1, pp. 271-278, 2014.

[28] N. Senroy, "Generator coherency using the Hilbert-Huang transform," IEEE Transactions on Power Systems, vol. 23, no. 4, pp. 1701-1708, 2008.

[29] B. P. Soni, A. Saxena, and V. Gupta, "A least square support vector machine-based approach for contingency classification and ranking in a large power system," Cogent Engineering, vol. 3, no. 1, p. 1137201, 2016.

[30] F. Hashiesh, H. E. Mostafa, A.-R. Khatib, I. Helal, and M. M. Mansour, "An intelligent wide area synchrophasor based system for predicting and mitigating transient instabilities," IEEE Transactions on Smart Grid, vol. 3, no. 2, pp. 645-652, 2012.

[31] C. Cortes and V. Vapnik, "Support vector machine," Machine Learning, vol. 20, no. 3, pp. 273-297, 1995.

[32] B. Soni, A. Saxena, and V. Gupta, "Support vector machine based approach for accurate contingency ranking in power system," in Proceedings of the 12th Annual IEEE India Conference (INDICON-2015), pp. 1-5, IEEE, Jamia Millia Islamia, New Delhi, India, December 2015.

[33] F. R. Gomez, A. D. Rajapakse, U. D. Annakkage, and I. T. Fernando, "Support vector machine-based algorithm for post-fault transient stability status prediction using synchronized measurements," IEEE Transactions on Power Systems, vol. 26, no. 3, pp. 1474-1483, 2011.

[34] P. M. Anderson and A. A. Fouad, Power System Control and Stability, John Wiley \& Sons, Hoboken, NJ, USA, 2008.

[35] Y. Li, W. Yuan, K. Chan, and M. Liu, "Coordinated preventive control of transient stability with multi-contingency in power systems using trajectory sensitivities," International Journal of Electrical Power \& Energy Systems, vol. 33, no. 1, pp. 147-153, 2011. 
[36] Powertech Labs Inc., Transient Security Assessment Tool (TSAT), Powertech Labs Inc., Surrey, BC, Canada V3W 7R7, 2002.

[37] B. P. Soni, A. Saxena, and V. Gupta, "A minimax polynomial approximation objective function approach for optimal design of power system stabilizer by embedding particle swarm optimization," Indonesian Journal of Electrical Engineering and Computer Science, vol. 14, no. 2, pp. 191-198, 2015.

[38] J. Kennedy, "Particle swarm optimization," in Encyclopedia of Machine Learning, pp. 760-766, Springer, New York, NY, USA, 2011.

[39] A. Saxena, B. P. Soni, and V. Gupta, "A chronological review and comparison of four evolutionary based algorithms," European Journal of Advances in Engineering and Technology, vol. 2, no. 1, pp. 35-41, 2015.

[40] B. P. Soni, A. Saxena, and V. Gupta, "Online identification of coherent generators in power system by using SVM," in Proceedings of the 2017 IEEE 4th International Conference on Power, Control \& Embedded Systems (ICPCES), pp. 1-5, Allahabad, India, March 2017.

[41] MathWorks, MATLAB: the Language of Technical Computing: Desktop Tools and Development Environment, Version 7, Vol. 9, MathWorks, Inc., Natick, MA, USA, 2005.

[42] R. D. Zimmerman, C. E. Murillo-Sánchez, and D. Gan, MATPOWER: A Matlab Power System Simulation Package, Manual, Vol. 1, Power Systems Engineering Research Center, Ithaca, NY, USA, 1997.

[43] F. Milano, "An open source power system analysis toolbox," IEEE Transactions on Power systems, vol. 20, no. 3, pp. 1199-1206, 2005. 


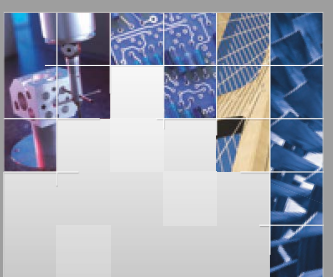

\section{Enfincering}
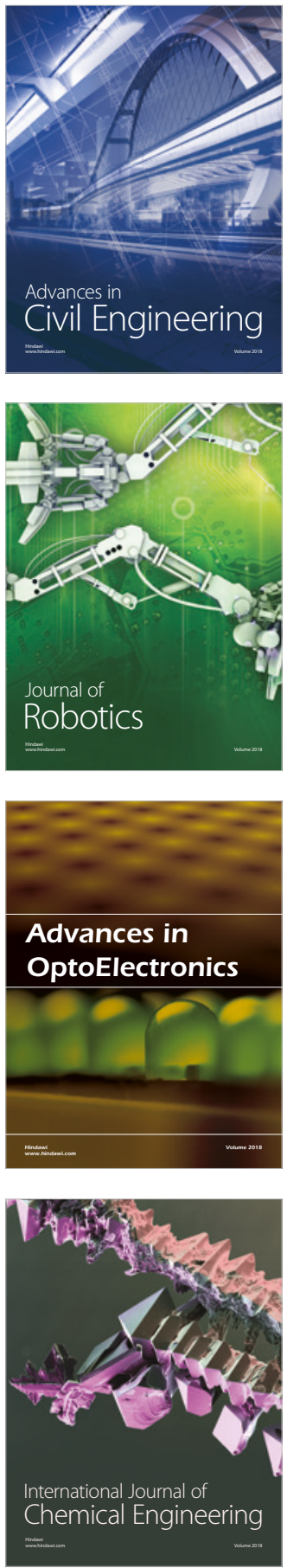

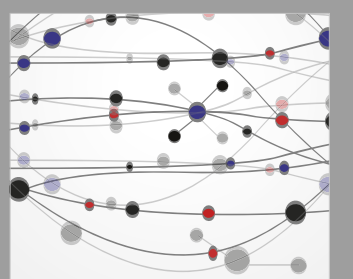

\section{Rotating \\ Machinery}

The Scientific World Journal

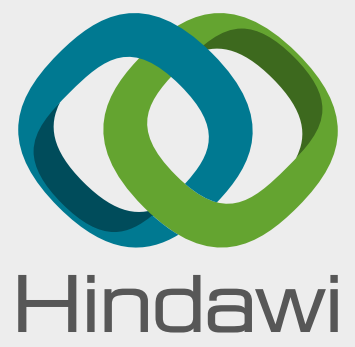

Submit your manuscripts at

www.hindawi.com
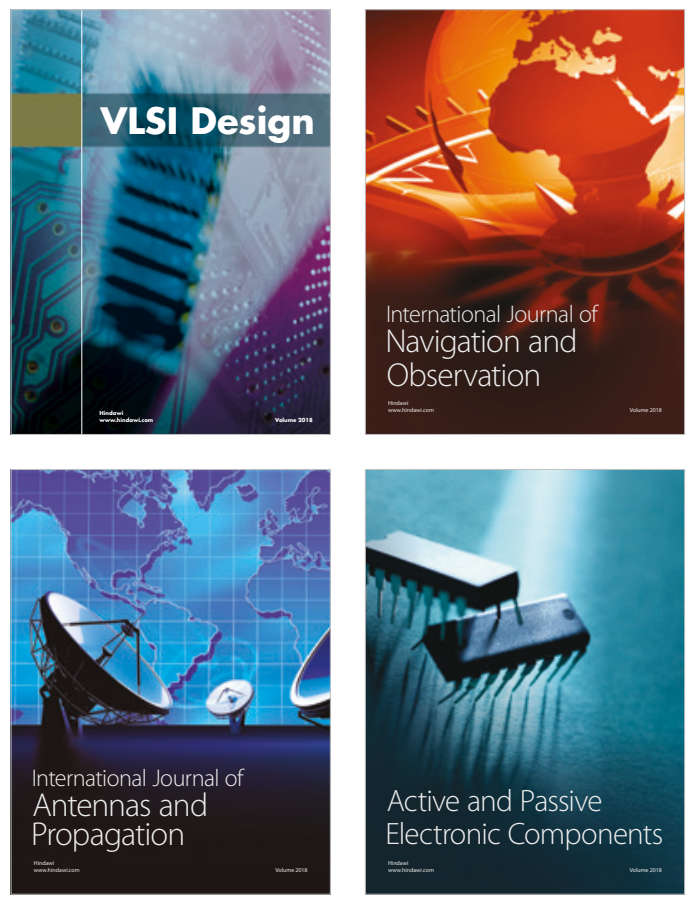
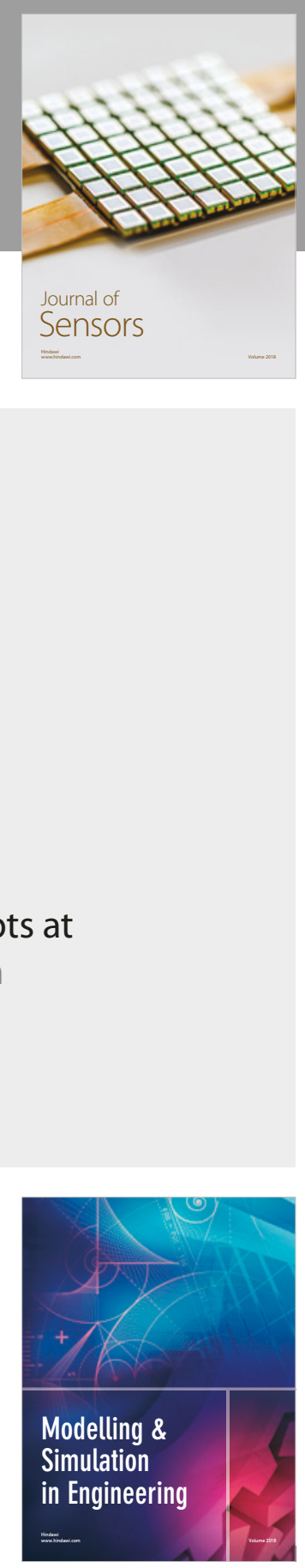

\section{Advances \\ Multimedia}
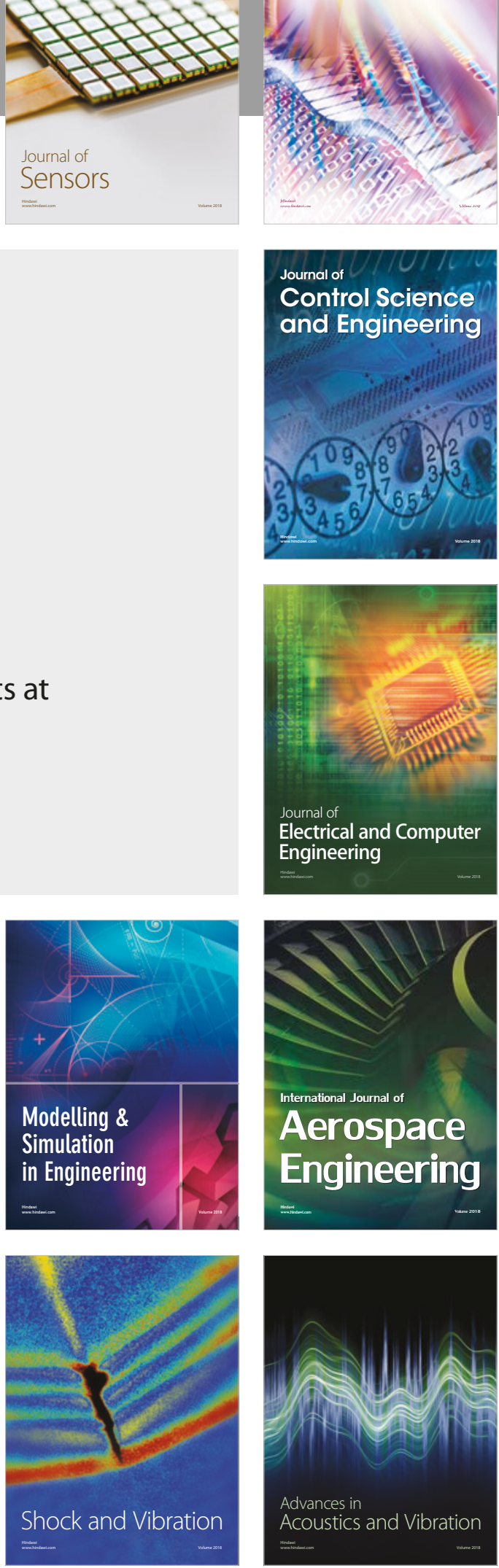